Rol de la investigación en el desarrollo regional del norte de La Paz. Páginas 287-308 en Revista de la Escuela de Ciencias de LA EduCACIÓn, AÑo 10, Número 9, enero a diciembre de 2014. ISSN 1851-6297. ISSN en Línea 23623349 .

\title{
ROL DE LA INVESTIGACION EN EL DESARROLLO REGIONAL DEL NORTE DE LA PAZ
}

\author{
Por Miguel Yucra Rojas (Universidad Mayor de San Andrés)*, Bolivia. \\ yucramiguel@yahoo.es \\ Fredy Enrique González (Universidad Pedagógica Experimental Libertador)**, \\ Venezuela. \\ fredygonzalez1950@gmail.com
}

Recibido: 14/06/2014 Aceptado: 01/09/2014

\section{Resumen}

El artículo versa sobre el crecimiento progresivo de la región amazónica del norte de La Paz, Bolivia, caracterizada por poseer una diversidad de recursos agroindustriales y que se ve atrapada en un estancamiento del desarrollo socioeconómico debido a la carencia de asistencia técnica, donde la población dedicada a la producción con poco valor agregado, permite débilmente la generación de ingresos temporales por la venta de productos, ocasionando postergaciones a sus aspiraciones de mejora a nivel local; por otra, la débil vinculación del gobierno departamental con la Universidad. En base a esa realidad, el artículo plantea la siguiente reflexión: ¿Cómo se podría contribuir para que el rol de la investigación coadyuvara al desarrollo regional del norte de La Paz? En respuesta, el artículo plantea un modelo de desarrollo de la amazonía del Norte de La Paz en base a la premisa de que las tendencias del desarrollo están relacionadas con la ciencia y tecnología, en coherencia a las directrices nacionales. Finalmente, se espera que el presente artículo contribuya a una reflexión institucional y que la propuesta presentada sea una referencia para quienes tienen el poder de decisión y de esta manera se pueda proyectar un desarrollo sustentable regional.

\section{Palabras Clave}

Investigación - Recursos - Vinculación - Amazonia.

* Estudiante del Doctorado en Gestion Universitaria, Magister en Desarrollo Sustentable, Diplomado en Gobernabilidad y Gerencia Política, Licenciado en Ingenieria Industrial (Universidad Mayor de San Andrés - UMSA). Investigador titular del Instituto de Investigaciones Industriales de la UMSA.

** Doctor en Educación. Formador de profesores de Matemática en la Universidad Pedagógica Experimental Libertador (UPEL, Núcleo Maracay, Estado Aragua, Venezuela). 
Revista de la Escuela de Ciencias de la Edducación, año 10, número 9, enero a diciembre de 2014. Páginas 287-308. ISSN 1851-6297. ISSN En LÍNEA 2362-3349. Rol DE LA INVESTIGACIÓN EN EL DESARROLLO REGIONAL DEL NORTE DE LA PAZ. Miguel Yucra Rojas - Fredy EnRique GonzÁlez.

\begin{abstract}
The article it turns on the progressive growth of the amazonian region of the north of La Paz, Bolivia, characterized for possessing a diversity of agroindustrial resources and that meets caught in a stagnation of the socioeconomic development due to the lack of technical assistance, where the population dedicated to the production with little added value, there allows weakly the generation of temporary income for the sale of products, causing postponements to his aspirations of improvement to local level; for other one, the weak entail of the departmental government with the University. On the basis of this reality, the article raises the following reflection: how might it contribute in order that the role of the investigation was contributing the regional development of the north of La Paz? In response, the article there raises a model of development of the amazonia of the north of La Paz on the basis of the premise of which the trends of the development are related to the science and technology, in coherence to the national directives. Finally, it hopes that the present article contributes to an institutional reflection and that the presented offer is a reference to those who have the power of decision and hereby it could project a sustainable regional development.
\end{abstract}

\title{
Key words
}

Research - Resources - Links - Amazonia.

\section{Introducción}

Bolivia tiene una superficie de $1.098 .581 \mathrm{~km} 2$, con 9 departamentos; al extremo norte se encuentra la región de la amazonia del departamento de La Paz, que comprende la provincia Abel Iturralde, cuyos municipios Ixiamas y San Buenaventura, con una superficie de 40.012 km2 y una población de 16.397 habitantes (INE, 2010), cuyos recursos maderables y no maderables entre otros. Dicho potencial proyecta una diversidad de emprendimientos agroindustriales orientado a la producción de productos alimenticios y no alimenticios.

La Nueva Constitución Política del Estado Plurinacional de Bolivia en el artículo 91 con relación a la educación superior, establece " La educación superior desarrolla procesos de formación profesional, de generación y divulgación de conocimientos orientados al desarrollo integral de la sociedad..."; así mismo, señala "La educación superior tiene por misión la formación integral de recursos humanos con alta calificación y competencia profesional; desarrollar procesos de investigación científica para resolver problemas de la base productiva y de su entorno social...".

Por su parte, la Ley de Educación aprobada en diciembre del 2010, en el artículo 28, establece que "la Educación Superior es el espacio educativo de formación profesional, de recuperación, generación y recreación de conocimientos y saberes, expresada en el desarrollo de la ciencia, la tecnología, la investigación y la innovación, que responde a las necesidades y demandas sociales, económicas, productivas y culturales de la sociedad y del Estado 
Revista de la Escuela de Ciencias de la Educación, año 10, número 9, enero a diciembre de 2014. Páginas 287-308. ISSN 1851-6297. ISSN en LÍNEA 2362-3349. ROL DE LA INVESTIGACIÓN EN EL DESARROLLO REGIONAL DEL NORTE DE LA PAZ. Miguel Yucra Rojas - Fredy EnRIQUe González.

Plurinacional". Asimismo en el artículo 52 establece que "La formación superior universitaria, es el espacio educativo de la formación de profesionales, desarrollo de la investigación científica-tecnológica, de la interacción social e innovación en las diferentes áreas del conocimiento y ámbitos de la realidad, para contribuir al desarrollo productivo del país expresado en sus dimensiones política, económica y sociocultural, de manera crítica, compleja y propositiva, desde diferentes saberes y campos de conocimiento en el marco de la Constitución Política del Estado Plurinacional".

El Estatuto Orgánico de la Universidad Mayor de San Andrés de 1988, manifiesta "la integración de la Universidad con la sociedad boliviana y la adecuación de la política universitaria a los intereses del pueblo boliviano", así también integra a la sociedad a través de planes y programas de carácter multidisciplinario e interdisciplinario y se compromete con el desarrollo socioeconómico del país, al mejoramiento de las condiciones de vida de sus habitantes; y contribuir al desarrollo regional con sentido de integración nacional".

Al relacionar las directrices de la Constitución Política del Estado, Ley de Educación y el Estatuto Orgánico de la UMSA; se deduce un común denominador respecto a la visión de la educación superior, la investigación científica-tecnológica, y el desarrollo integral de la sociedad; cuyos mecanismos disponibles, a la fecha no están siendo aplicados de manera institucionalizada en dichos ámbitos. Es decir, la presencia de la Universidad en dicha región hasta la fecha ha generado un efecto poco significativo respecto a la asistencia técnica y asesoramiento, a pesar de contar con un programa de desconcentración universitaria; por su parte, el Gobierno mediante sus representaciones municipales ha demostrado escasa vinculación con la Universidad para reforzar dicha actividad, donde la priorización de inversiones hacia proyectos productivos en la fase de planificación son considerados de segunda importancia, consecuentemente dichas prioridades se traducen en inversiones para infraestructura de construcciones civiles.

En la actualidad existe un amplio consenso sobre los efectos que ejerce la disponibilidad de recursos naturales y su intermediación comercial, sobre el crecimiento económico local y su sostenibilidad en el tiempo. Desde el punto de vista de la agroindustria, algunos autores han señalado que la inversión en investigación tiene un impacto de desarrollo. Dentro de los principales desafíos que enfrenta la investigación se destaca el de disponer de un adecuado mecanismo que facilite la realización de la misma, que en el caso de no realizarse podría seguir perdurando la interrogante ¿Cuál es el rol de la investigación en el desarrollo de la región amazónica?

Para el cual se ha abordado desde el análisis regional, las directrices del gobierno y la universidad, el plan nacional de desarrollo, la vinculación e interacción entre la universidad y el gobierno departamental; a partir de la cual se propone un plan de acciones traducidos en: creación de un Vicerrectorado, 
Revista de la Escuela de Ciencias de la Educación, año 10, número 9, enero a diciembre de 2014. Páginas 287-308. ISSN 1851-6297. ISSN EN LINEA 2362-3349. ROL DE LA INVESTIGACIÓN EN EL DESARROLLO REGIONAL DEL NORTE DE LA PAZ. Miguel Yucra Rojas - Fredy EnRIQue González.

institucionalización de la vinculación UMSA-Gobierno, fortalecimiento tecnológico de la carrera de Ingeniería Industrial Amazónica y la reorientación de los fondos IDH, para concluir presentando algunas conclusiones; que podrían ser consideradas en la toma de decisiones de las autoridades correspondientes tanto de la universidad como del gobierno, considerando la visión de generar ciencia y tecnología que contribuya al desarrollo regional del norte de La Paz.

\section{Análisis de la región amazónica del norte de La Paz}

El Plan de Desarrollo del Departamento de La Paz al 2020, señala que la región del norte amazónico del Departamento, es un potencial importante para la producción forestal, donde el $39,46 \%$ de sus tierras son aptas para el uso forestal, cuya utilización de recursos naturales condicionado al cumplimiento de las recomendaciones de uso sostenible, permitiría una oportunidad de aprovechamiento de la madera y productos agroforestales como la goma, castaña, palmito, plantas medicinales entre otros. Actualmente, en la región se extrae madera, especialmente en el área de Ixiamas y San Buenaventura. Por otro lado, el $15,09 \%$ de las tierras de dichas regiones son praderas aptas para el uso ganadero extensivo tropical de bovinos y sujeto a condicionantes de uso a través de la aplicación de prácticas de cuidado y conservación. La actividad ganadera se constituye en una visión de una región desarrollada, especialmente en las áreas de Palos Blancos (Alto Beni) y las llanuras de Ixiamas.

Los recursos potenciales en la amazonia del norte de La Paz son: maderables (mara, cedro, roble, aliso, mara macho, almendrillo, bibosi, mapajo, ocho, palo maría, verdolaga, laurel) y no maderables (castaña, goma, cacao silvestre, majo, jatata), productos agrícolas (arroz, maíz, plátano, cítricos, frejol, verduras, hortalizas, walusa, camote, palta, mango, coco, maní, yuca, frutas, flores y caña de azúcar), turismo, potencial piscícola, apícola, ganadero, aves, entre otros.

Como se puede apreciar la región amazónica presenta una diversidad de oportunidades para la creación de emprendimientos agroindustriales, tomando como referencia el potencial de recursos existentes y transformar en productos tales como harina de arroz, arroz pre cocido, aceites de majo, leche de majo, carne deshidratada o ahumada de surubí, sábalo, pacú; muebles, casas prefabricadas, artesanías, industrialización del copoazu, achachairu, entre algunos como referencia. La experiencia agroindustrial de la región amazónica, sobre la transformación de productos primarios en productos con valor agregado a escala industrial, se podría considerar insuficiente, pues debido a la escasa existencia de asesoramiento técnico a nivel local, que permitan facilitar la generación de emprendimientos productivos, esta carencia obliga al productor local a estancar sus aspiraciones de mejora de ingresos, favoreciendo a los intermediarios comerciantes para la compra de las materias primas, muchas veces a precios por debajo de los costos de producción; al mismo tiempo pos- 
Revista de la Escuela de Ciencias de la Educación, año 10, número 9, enero a diciembre de 2014. Páginas 287-308. ISSN 1851-6297. ISSN en LÍNEA 2362-3349. ROL DE LA INVESTIGACIÓN EN EL DESARROLLO REGIONAL DEL NORTE DE LA PAZ. Miguel Yucra Rojas - Fredy EnRIQUe González.

tergando las aspiraciones de educación superior de sus hijos e incrementando la migración a los centros urbanos de otros departamentos.

Al respecto, ¿de qué sirve la disponibilidad de riqueza natural si aun se percibe que las acciones de asistencia técnica, asesoramiento y la presencia de la UMSA están débiles en la región amazónica del norte de La Paz?; entrevistas con autoridades y representaciones locales expresaron que el estancamiento se atribuyen a: a) planificación realizada sin participación local, b) la visión centrada en la autoridad municipal de turno, c) mayor priorización de inversión en infraestructura, d) poca autonomía en la decisiones locales, e) planificación en función de interese políticos, f) rendición de cuentas por exigencia comunitaria, g) cambios de personal técnico, h) poca alianza institucional, i) la función de Alcaldes orientada a lo operativo.

Por lo tanto, ante las anomalías y oportunidades, se puede visibilizar la necesidad urgente de una orientación científica y financiera para revertir las incoherencias y generar desarrollo en el marco de una contribución institucional de la Universidad, cuyo rol debiera ser traducido en el desarrollo de la región amazónica del norte de La Paz.

\section{Directriz nacional}

La Nueva Constitución Política del Estado Plurinacional de Bolivia (CPE), favorece la construcción de un Estado basado en el respeto e igualdad entre todos, con principios de soberanía, dignidad, complementariedad, solidaridad, armonía y equidad en la distribución y redistribución del producto social, donde predomine la búsqueda del vivir bien; con respeto a la pluralidad económica, social, jurídica, política y cultural de los habitantes de esta tierra; en convivencia colectiva con acceso al agua, trabajo, educación, salud y vivienda para todos.

En el capítulo VIII AMAZONIA, artículo 390, parágrafo I, de la CPE, establece que la cuenca amazónica boliviana constituye un espacio estratégico de especial protección para el desarrollo integral del país por su elevada sensibilidad ambiental, biodiversidad existente, recursos hídricos y por las ecoregiones. Por otra parte, la política del Estado Plurinacional con relación a la educación superior está respaldada por la CPE, que en su artículo 91 parágrafo I, declara " La educación superior desarrolla procesos de formación profesional, de generación y divulgación de conocimientos orientados al desarrollo integral de la sociedad..."; así mismo, el parágrafo Il del mismo artículo, señala "La educación superior tiene por misión la formación integral de recursos humanos con alta calificación y competencia profesional; desarrollar procesos de investigación científica para resolver problemas de la base productiva y de su entorno social ...". Respecto a ciencia, tecnología e investigación; la CPE en su artículo 103, parágrafo III, señala "El Estado, las universidades, las empresas productivas y de servicios públicas y privadas, y las naciones y pueblos indígena originario campesinos, desarrollaran y coordinaran procesos de investigación, innovación, 
Revista de la Escuela de Ciencias de la Educación, año 10, número 9, enero a diciembre de 2014. Páginas 287-308. ISSN 1851-6297. ISSN EN LINEA 2362-3349. ROL DE LA INVESTIGACIÓN EN EL DESARROLLO REGIONAL DEL NORTE DE LA PAZ. Miguel Yucra Rojas - Fredy EnRIQue González.

promoción, divulgación, aplicación y transferencia de ciencia y tecnología para fortalecer la base productiva e impulsar el desarrollo integral de la sociedad"

\section{Plan nacional de desarrollo}

El Plan Nacional de Desarrollo "Bolivia digna, soberana, productiva y Democrática para vivir bien" establece que la Ciencia, Tecnología e Innovación son los instrumentos fundamentales para el desarrollo de los sectores productivos donde está incluido el industrial. Enfatiza que la ciencia y tecnología en el sector gubernamental se ha presentado de forma individualizada y muchas veces no coordinada entre sus diferentes ministerios, mediante la creación de proyectos con base tecnológica y con el Viceministerio de Ciencia y Tecnología.

Por su parte, la Ley No. 070 Educación de Bolivia, en el artículo 52 establece que "La formación superior universitaria, es el espacio educativo de la formación de profesionales, desarrollo de la investigación científica-tecnológica, de la interacción social e innovación en las diferentes áreas del conocimiento y ámbitos de la realidad, para contribuir al desarrollo productivo del país expresado en sus dimensiones política, económica y sociocultural, de manera crítica, compleja y propositiva..."

El Dialogo Departamental "Pacto por La Paz" realizado en la ciudad de La Paz-Bolivia los días 17, 18 y 19 de junio de 2013; facilitó la discusión y el consenso de los participantes respecto a la identificación de estrategias, políticas y compromisos institucionales, permitiendo la construcción del futuro del departamento de La Paz. El documento elaborado por el Viceministerio de Ciencia y Tecnología del 2012, en el componente Transformación Industrial y Manufacturera, señala como objetivo el de fomentar la investigación y generación de conocimiento hacia áreas estratégicas del sector de Transformación Industrial y Manufacturera para el desarrollo del país.

Dando seguimiento a dichas iniciativas, este organismo sectorial dependiente del Ministerio de Educación, organizó el "Encuentro Nacional hacia la Soberanía Científico - Tecnológica", realizado el 21 y 22 de octubre de 2013, con la participación de representantes de universidades, instituciones y empresas públicas y privadas, organizaciones sociales, campesinas, indígenas y originarias; generándose como resultado líneas estratégicas respecto al componente: Estado de la innovación tecnológica para la producción, industrialización, diversificación y generación de riqueza; tales como: a) Fortalecer la interacción gobierno, academia, sectores productivos y sociales que favorecen la innovación tecnológica, b) Promover y crear Centros de Innovación, Centros de Investigación, Unidades de Transferencia de Tecnología, Incubadoras de Empresas, desde el Gobierno, las Universidades y Sector productivo.

El compromiso social proyectado para el desarrollo del país y especialmente del departamento de La Paz, no ha prosperado en relación a la vinculación, formación de cuadros especializados y la ejecución de estrategias de 
Revista de la Escuela de Ciencias de la Educación, año 10, número 9, enero a diciembre de 2014. Páginas 287-308. ISSN 1851-6297. ISSN en LÍNEA 2362-3349. ROL DE LA INVESTIGACIÓN EN EL DESARROLLO REGIONAL DEL NORTE DE LA PAZ. Miguel Yucra Rojas - Fredy EnRIQUe González.

desarrollo nacional y regional; que podría atribuirse al protagonismo individual e institucional existente entre el gobierno y la universidad, motivado a la escasa interacción para compartir los recursos asignados y realizar una planificación conjunta con visión de región y país.

La Universidad mediante sus unidades académicas a pesar de participar en eventos institucionales con el gobierno de manera circunstancial, no ha logrado calar espacios de incidencia, a raíz de los círculos poco accesibles del gobierno a recibir propuestas o al temor de compartir los recursos económicos, propiciado el crecimiento de compromisos protagónicos y aumentando la brecha ya existente de acercamiento entre ambas instituciones. Entonces, ¿dónde está el deseo de desarrollar las regiones y fortalecer la capacidad tecnológica en las provincias, cuando seguimos enclaustrados de promesas para las formaciones en las regiones del interior del país, o en su caso, cuando se crean las carreras y se deja al olvido la formación tecnológica de dichos aspirantes universitarios, sin equipamiento, sin bibliotecas, sin ejercicios de investigación, sin relación del pregrado con el postgrado?

\section{La vinculación entre la UMSA y el gobierno}

El Estatuto Orgánico de la Universidad Mayor de San Andrés (UMSA) de 1988, manifiesta "la integración de la Universidad con la sociedad boliviana y la adecuación de la política universitaria a los intereses del pueblo boliviano, así también integra a la sociedad a través de planes y programas de carácter multidisciplinario e interdisciplinario y se compromete con el desarrollo socioeconómico del país, al mejoramiento de las condiciones de vida de sus habitantes; y contribuir al desarrollo regional con sentido de integración nacional".

El Plan Estratégico Institucional 2012-2016 de la UMSA, plantea como objetivo estratégico "alcanzar el conocimiento técnico y científico de los profesionales docentes, investigadores y estudiantes partícipes de proyectos y de las sociedades científicas". Si consideramos algunas políticas establecidas como: a) Política de incentivo para la investigación y el desarrollo; b) Política de relacionamiento entre la investigación y el desarrollo con el pre y postgrado. Sin embargo, la UMSA a pesar de contar con alrededor de 38 Institutos de Investigaciones en distintas áreas, entre ellas hidráulica, metalurgia, ambiental, industrial, procesos químicos, agrícola y mecánica, y que representan un potencial generador de ciencia y tecnología para la región amazónica del norte de La Paz; a la fecha, aun no ha surtido su efecto en dicha región. Los esfuerzos realizados por cada unidad académica en pretender llegar al interior del departamento de La Paz, gira alrededor de las convocatorias a los fondos concursables IDH que anualmente realiza el Departamento de Investigación, Postgrado e Interacción Social (DIPGIS), cuyas propuestas en su mayoría nacen de las experiencias o ideas personales de cada investigador, desvirtuando la visión de pertinencia para el desarrollo nacional. 
Revista de la Escuela de Ciencias de la Educación, año 10, número 9, enero a diciembre de 2014. Páginas 287-308. ISSN 1851-6297. ISSN EN LINEA 2362-3349. ROL DE LA INVESTIGACIÓN EN EL DESARROLLO REGIONAL DEL NORTE DE LA PAZ. Miguel Yucra Rojas - Fredy EnRIQue González.

A esto se suma la inexistencia institucionalizada de mecanismos al interior de la UMSA para la integración del pregrado y el postgrado, consecuentemente las políticas establecidas en el plan institucional aun están sin efectos, tanto a nivel urbano y mucho mas a nivel de las provincias, donde existen carreras descentralizadas, tal es la carrera de Ingeniería Industrial Amazónica que en su momento fue creada para generar desarrollo regional; que sin embargo, la poca atención brindada por instancias superiores hace que dichos resultados esperados relacionados con la investigación, el desarrollo y la "Generación de Ciencia y Tecnología en la UMSA" se van disolviendo en simplemente protagonismos circunstanciales de cada autoridad de turno a nivel académico.

\section{Interacción UMSA-Gobierno departamental en el desarrollo de la región norte de La Paz}

Engloba como desarrollo a la satisfacción de necesidades básicas humanas de carácter material y aquellas intangibles, como son la satisfacción de necesidades de expresión, creatividad e igualdad, así como las condiciones de convivencia, permitiéndoles comprender y orientar sus propios destinos (Hidalgo, 2004).

El desarrollo si es autentico inevitablemente debe ser a escala humana, motivo por el cual el desarrollo se refiere a las personas y no a los objetos, el mejor proceso de desarrollo será aquél que permita elevar más la calidad de vida de las personas y la calidad de vida dependerá de las posibilidades que tengan las personas de satisfacer adecuadamente sus necesidades humanas fundamentales (PNUD, 2001).

Un concepto universal coherente a entender es que el DESARROLLO es un DERECHO HUMANO y que como tal, el fortalecimiento de la sociedad civil es un mecanismo de involucramiento planificado en los escenarios de participación en los distintos niveles de acciones y decisiones de los países (Declaración Universal de los Derechos Humanos, 1948).

Las perspectivas de desarrollo contraídas en la Sede de las Naciones Unidas en Nueva York del 6 al 8 de septiembre el año 2000, los Jefe de Estado y de Gobierno así como las altas autoridades de 189 países, incluida Bolivia, que asistieron a la llamada Cumbre del Milenio de las Naciones Unidas, aprobaron la Declaración del Milenio, en la que se establecieron los Objetivos y Metas del Milenio; se podría decir que el resultado es escaso a nivel de país y especialmente en la región amazónica del norte de La Paz, puesto que Los Objetivos de Desarrollo del Milenio caducan en el 2015; al respecto la $37^{a}$ reunión de la Conferencia General de la UNESCO, fijó el rumbo del trabajo mediante la nueva Estrategia a Plazo Medio (2014-2021), uno de los documentos adoptados por la Conferencia, posiciona a la UNESCO en un papel líder en la definición de los objetivos de desarrollo que sustituirán a los Objetivos de Desarrollo del Milenio en los ámbitos de la educación, la ciencia, la cultura y la comunicación e infor- 
Revista de la Escuela de Ciencias de la Educación, año 10, número 9, enero a diciembre de 2014. Páginas 287-308. ISSN 1851-6297. ISSN EN LINEA 2362-3349. RoL DE LA INVESTIGACIÓN EN EL DESARRolLo REGIONAL DEL NORTE DE LA PAZ. Miguel Yucra Rojas - Fredy EnRIQUe González.

mación; por lo que actualmente Naciones Unidas y otros foros internacionales están debatiendo para fijar una nueva agenda del desarrollo.

En Bolivia muchas veces olvidamos el valor humano y consideramos que lo económico es lo mas imprescindible, donde muchas veces el distribuir la riqueza en efectivo puede resolver el momento de la crisis, que sin embargo, no es sostenible en el tiempo; los criterios anteriores respecto al desarrollo y el valor humano deben ser tomados en cuenta en todo momento de la planificación. Seguramente nuestro país nuevamente asumirá los compromisos y que esta reflexión pueda alertar a la Universidad y el Gobierno en considerar a pertinencia entre las demandas sociales y los proyectos de investigación.

Por otra parte, el artículo publicado en febrero de 2014, plantea que "la interacción de la universidad con el gobierno sea nacional, departamental, municipal o comunal debe cerrar un ciclo, donde la universidad le informa al gobierno sobre el avance de la ciencia y la tecnología en diversos campos de interés nacional; en retorno el gobierno le hace conocer sus requerimientos de estudios en diferentes campos de acción social y le da a la universidad los dineros y el financiamiento necesarios para desarrollar esos estudios" (Escalera, 2014).

A todo esto, la relación entre la UMSA y el Gobierno a nivel Departamental y Nacional aun no ha logrado consolidarse de manera institucionalizada, continúa en una fase de experimentación interinstitucional. Sin embargo, las experiencias directas con algunos gobiernos municipales fueron con mayor aceptación, prueba de ello muchos de los municipios del departamento de La Paz se han favorecido con la asistencia y elaboración de proyectos específicos. Esto fue posible a raíz que algunas unidades académicas como el Instituto de Investigaciones Industriales han llevado la iniciativa, razón por la cual se podría traducir en un encuentro tibio y circunstancial. Sin embargo, se considera que la vía no es la correcta ni sostenible, por lo que urge organizar un mecanismo que viabilice la vinculación institucionalizada al interior de cada institución para mantener una interacción sustentable.

\section{Modelo de desarrollo del norte de La Paz}

Austin (1987) define a la agroindustria como la actividad que engloba el manejo, preservación y transformación de materias primas provenientes de la agricultura, la ganadería, el sector forestal y el pesquero, todo ello enmarcado en el sistema agroalimentario. Esta actividad económica comprende la producción, industrialización y comercialización de productos agrarios, pecuarios, forestales y biológicos.

Lo señalado anteriormente, constituye una oportunidad para proponer una opción de modelo de desarrollo agroindustrial para la región amazónica del norte de La Paz, si promovemos la investigación agroindustrial en torno a una vinculación institucionalizada entre la UMSA y el Gobierno. Este modelo se 
Revista de la Escuela de Ciencias de la Educación, año 10, número 9, enero a diciembre de 2014. Páginas 287-308. ISSN 1851-6297. ISSN en LÍNEA 2362-3349. ROL DE LA INVESTIGACIÓN EN EL DESARROLLO REGIONAL DEL NORTE DE LA PAZ. Miguel Yucra Rojas - Fredy EnRIQue González.

sustenta en la potencialidad de riqueza natural existente en la región, a la cual se deberá complementar el concurso de asistencia técnica y asesoramiento por medio de la Universidad y sumar la participación del Gobierno con la asignación de recursos financiaros. Es decir, para que sea funcional el modelo de desarrollo se requiere al menos cuatro aspectos: a) la participación de la Universidad con investigación tecnológica; b) la disponibilidad de recursos naturales; c) la participación de los actores locales; d) la participación del gobierno con decisión política e inversiones.

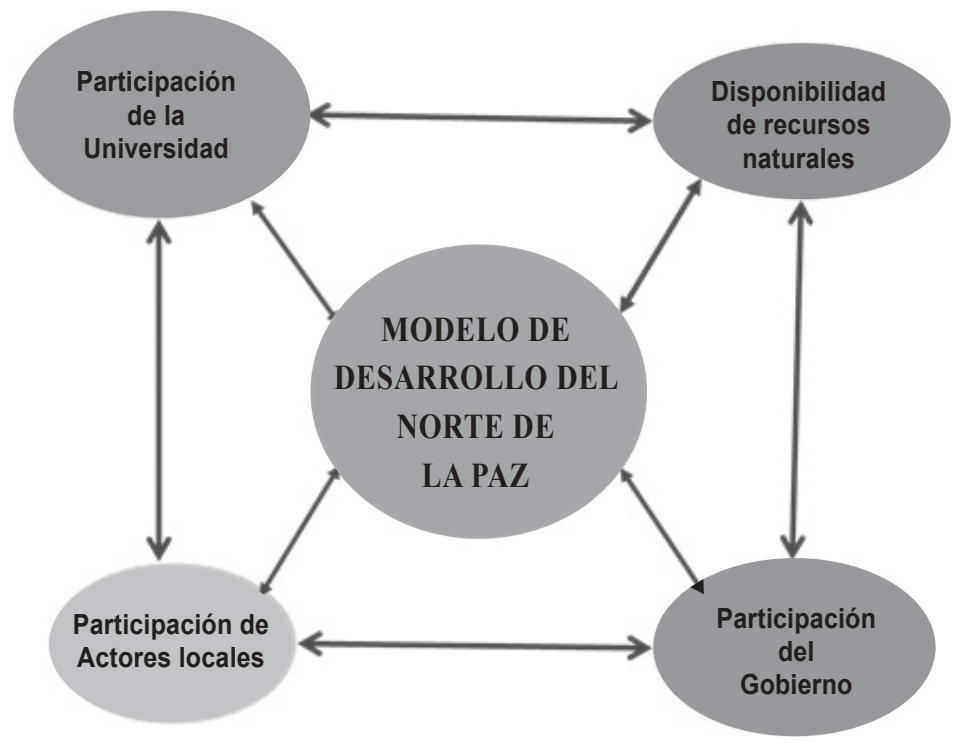

Esquema 1: Integración de recursos para el desarrollo

\section{a) Participación de la Universidad con investigación tecnológica}

En el departamento de La Paz, la Universidad Mayor de San Andrés constituye la oferta académica de formación pública en la que cuenta con 38 Institutos de investigación, entre ellas de carácter social, económico, ambiental, tecnológico; podrían reformular su enfoque de investigación considerando la demanda social y la disponibilidad de los recursos naturales; para el cual será necesario la desconcentración racionalizada y especializada por prioridad sectorial de sus áreas académicas en coordinación con el gobierno.

\section{b) Disponibilidad de recursos naturales}

La región amazónica del norte de La Paz, es un potencial agroindustrial, que en lugar de ser desmantelada la materia prima por comerciantes; el Gobierno deberá concentrar su esfuerzo para implementar unidades productivas en base a la investigación tecnológica que la Universidad oferte. 
Revista de la Escuela de Ciencias de la Educación, año 10, número 9, enero a diciembre de 2014. Páginas 287-308. ISSN 1851-6297. ISSN en LÍNEA 2362-3349. ROL DE LA INVESTIGACIÓN EN EL DESARRolLo REGIONAL DEL NORTE DE LA PAZ. Miguel Yucra Rojas - Fredy EnRIQue González.

\section{c) Participación de los actores locales}

Existe la voluntad de los actores locales para coadyuvar la implementación de proyectos; sin embargo, no existe una planificación sostenible por sus autoridades; al respecto tanto la Universidad como el Gobierno deben incluir a los actores locales desde la priorización, planificación, ejecución e implementación de cada uno de los proyectos sectoriales, a fin de garantizar la sustentabilidad institucional.

\section{d) Participación del gobierno}

El Gobierno con la llave de recursos financieros y con decisión política, puede fomentar las inversiones tanto para la fase de investigación, coadyuvar las acciones de formación especializada, la actividad investigativa y la implementación de los emprendimientos productivos.

Por lo tanto, no es posible generar desarrollo regional si al menos los aspectos identificados están fuera de su involucramiento, eso significa que las autoridades con poder de decisión especialmente de la Universidad y el Gobierno se despojen de protagonismos individuales y encaminen con propuestas realizables en torno a una visión de país y considerando a la región amazónica como un sujeto que requiere soluciones y no como un objeto de estudio que hasta la fecha se viene considerando.

Para consolidar esa integración, es prioritario mantener una vinculación institucional considerando la directriz nacional, la misma que se esquematiza a continuación:

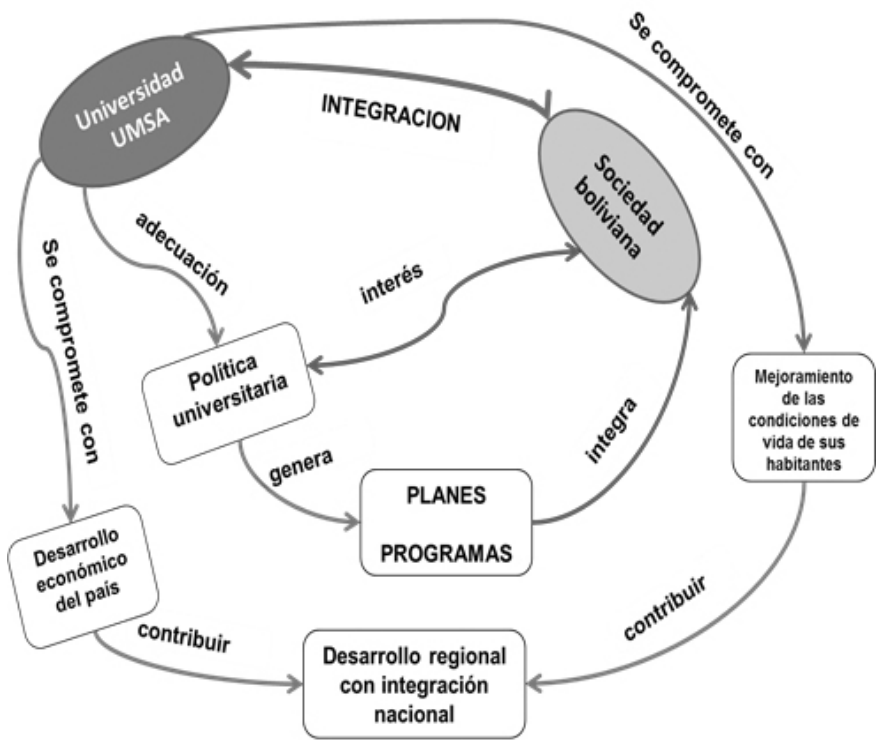

Esquema 2: Vinculación de la Universidad al desarrollo regional 
Revista de la Escuela de Ciencias de la Educación, año 10, número 9, enero a diciembre de 2014. Páginas 287-308. ISSN 1851-6297. ISSN EN LINEA 2362-3349. ROL DE LA INVESTIGACIÓN EN EL DESARROLLO REGIONAL DEL NORTE DE LA PAZ. Miguel Yucra Rojas - Fredy EnRIQue González.

El esquema 2, ha sido construido en base a la directriz académica de la Universidad Mayor de San Andrés que deberá estar comprometida con el ideal de mejorar las condiciones de vida de los habitantes, de integrar a la sociedad boliviana, de adecuar las políticas universitarias y comprometido con el desarrollo económico del país (directriz nacional), todo ello orientado al desarrollo regional; el cual deberá ser plasmado en acciones reales, con investigación, con involucramiento de los actores locales, con formación del capital especializado y con inversiones, que en el esquema 1 se ha visibilizado. Al respecto se plantea el plan de acción desde el pensamiento académico para fortalecer la actividad investigativa.

\section{Plan de acción para el desarrollo en la región amazónica del norte de La Paz}

La región amazónica del norte de La Paz puede revertir su estancamiento de desarrollo si incursionamos a la oportunidad de uso del conocimiento tradicional regional y una orientación académica con apoyo financiero desde las instancias del Gobierno y la Universidad. Al respecto, a fin de llevar la iniciativa académica se plantea cuatro acciones que faciliten la actividad tecnológica en la región amazónica del norte de La Paz: a) Creación del Vice-Rectorado de investigación y postgrado; b) Institucionalización de la vinculación UniversidadGobierno; c) Necesidad de fortalecimiento tecnológico a la Carrera de Ingeniería Industrial Amazónica en San Buenaventura; d) Reorientación en el uso de los fondos IDH. Los mismos que son desglosados a continuación:

\section{a) Creación del Vice-Rectorado de investigación y postgrado}

El manual de Organización y Funciones de la UMSA del 2008, establece once funciones para el Vicerrectorado de la UMSA, de las cuales sustraemos textualmente dos funciones: 7. Dirigir las políticas académicas, investigación, interacción social y postgrado emanadas del Gobierno Universitario; 9. Orientar el proceso de formulación, seguimiento y ejecución del nuevo modelo académico para el siglo XXI.

En el mismo documento, se establece nueve funciones para la Jefatura de Departamento Investigación, Postgrado e Interacción Social (DIPGIS), de las cuales consideramos para efectos de comparación la función 3. Planificar, orientar, evaluar, coordinar entre los niveles de decisión y ejecución, llevar adelante las políticas de Investigación, Postgrado e Interacción Social.

Contrastando las funciones del Vicerrectorado y el DIPGIS respecto a la Investigación, Postgrado e Interacción Social, se concluye en cierta dualidad de funciones, afectando una especificidad de pertinencia académica, cuando se considera en otras experiencias Universitarias fuera de Bolivia, al Vicerrectorado como una función de gestión y no operativa; a esto se puede adicionar como otro fundamento un extracto de la función nueve "nuevo modelo académico para 
Revista de la Escuela de Ciencias de la Educación, año 10, número 9, enero a diciembre de 2014. Páginas 287-308. ISSN 1851-6297. ISSN EN LINEA 2362-3349. RoL DE LA INVESTIGACIÓN EN EL DESARRolLO REGIONAL DEL NORTE DE LA PAZ. Miguel Yucra Rojas - Fredy EnRIQUe González.

el siglo XXI", esto le afianza una visión para la creación de un Vicerrectorado de Investigación y Postgrado, que ese nuevo modelo académico podría dar un fruto al nivel de otras Universidades.

Como un ejemplo, se presenta a la Universidad Pedagógica Experimental Libertador (UPEL) de Venezuela, donde la coordinación de la función docente de pregrado y de postgrado, corresponda a dos Vicerrectorados diferentes; el Vicerrectorado de Docencia y el Vicerrectorado de Investigación y Postgrado. Entre algunas políticas principales del Vicerrectorado de Investigación y Postgrado, podemos destacar:

- Desarrollar programas de postgrado con base a criterios de pertinencia social e integración

- Promover, difundir y divulgar la producción de investigaciones.

- Valorar la calidad y pertinencia del quehacer académico y de investigación.

- Generar proyectos que respondan a la realidad comunitaria y sus procesos de cambio y transformación.

Como se puede observar el ejemplo de la UPEL, está relacionado con el postgrado, integración, investigación, difusión, investigación en pregrado, pertinencia, respuesta a la demanda social y adecuación a procesos de cambio. Es decir, es una experiencia que nos lleva la delantera respecto a aceptar los cambios tecnológicos y académicos. Por lo tanto, la UMSA tiene la oportunidad de iniciar una transformación institucional debido al crecimiento de estudiantes a nivel de pregrado, postgrado, demanda de investigación con pertinencia social, y desarrollar una gestión académica eficiente y eficaz; a través de una reorganización administrativa, de investigadores y servicios tecnológicos; a su vez se podría incorporar como una función principal del Vicerrectorado la gestión de la investigación apoyado en un Modelo de Gestión de la Investigación en la UMSA construido a partir del ser y el deber ser de cada uno de los institutos de investigación existentes, con la cual se pueda coordinar y ejecutar la visión de vinculación, integración, desarrollo regional, interacción social, para desarrollar la investigación con pertinencia social y académica.

\section{b) Institucionalización de la vinculación Universidad-Gobierno}

La interacción de la universidad con el gobierno sea nacional, departamental, municipal o comunal debe cerrar un ciclo, donde la universidad le informa al gobierno sobre el avance de la ciencia y la tecnología en diversos campos de interés nacional; en retorno el gobierno le hace conocer sus requerimientos de estudios en diferentes campos de acción social y le da a la universidad los dineros y el financiamiento necesarios para desarrollar esos estudios (Escalera, 2014).

La UMSA con más de 70.000 estudiantes en pregrado, con 54 carreras y 38 institutos de investigación constituye un potencial de capital humano, donde sus profesionales están brindando servicios en distintas instituciones públicas 
Revista de la Escuela de Ciencias de la Educación, año 10, número 9, enero a diciembre de 2014. Páginas 287-308. ISSN 1851-6297. ISSN EN LINEA 2362-3349. ROL DE LA INVESTIGACIÓN EN EL DESARROLLO REGIONAL DEL NORTE DE LA PAZ. Miguel Yucra Rojas - Fredy EnRIQue González.

y privadas; situación que amerita consolidar una interacción sostenible entre el gobierno y la Universidad, con la finalidad de responder los requerimientos de estudios en diferentes campos de acción social, cuya infraestructura académica debería ser aprovechada al máximo por las instancias gubernamentales a distintos niveles; sin embargo, ocurre lo contrario ambos van por vías diferentes distrayendo muchas veces a los actores y consecuentemente desperdiciando las inversiones tanto nacionales como de cooperación internacional, por esa brecha existente a un trabajo en equipo institucional.

Muchas veces no comprendemos que los recursos financieros generados por el país, es producto del concurso de la comunidad boliviana y por lo tanto se debería promover una distribución de dicha riqueza no en efectivo, sino mediante inversiones que posibiliten la generación de empleo e ingresos, posiblemente con esta modalidad podamos de manera formal disminuir la pobreza. Por su parte la UMSA, debería actuar como un socio tecnológico desde lo urbano hasta las regiones más necesitadas como es la región amazónica del norte de La Paz, donde las condiciones y posibilidades de formación superior son precarias y las respuestas de las altas direcciones académicas no llegan.

Una experiencia de pregrado con apoyo del autor del presente artículo dependiente del Instituto de Investigaciones Industriales de la UMSA y con la finalidad de coadyuvar el desarrollo local se proyectó un Centro Tecnológico Agroindustrial (CTA), orientado a la investigación tecnológica y al desarrollo productivo de la agroindustria a nivel regional, localizado en el municipio de San Buenaventura para brindar el servicio a dicha región; cuya estructura funcional referida a tres unidades con sus áreas respectivas:

- Unidad de Investigación y Desarrollo; para atender las necesidades de los productores de la región en toda la cadena agroindustrial, investigando y desarrollando mejoras en las etapas de cultivo y procesos de producción; cuyas áreas: Investigación y desarrollo de nuevos productos, Investigación y desarrollo de producción agroindustrial, preparación y evaluación de proyectos, Servicios de Laboratorio.

- Unidad de Información y Capacitación; proporcionara información precisa y con valor añadido sobre todas aquellas áreas de interés agroindustrial y tecnológico que puedan repercutir en una mejora de la competitividad del sector agroindustrial; cuyas áreas: Información, capacitación.

- Unidad de Tecnología de Procesos; pretende atender las necesidades de los productores de la región en el aspecto industrial, desarrollando nuevos productos y procesos mediante tecnologías limpias enfocadas en el uso sustentable de los recursos naturales y protección ambiental; cuyas áreas: Desarrollo industrial de procesos y productos, control de calidad y seguridad de los productos, medio ambiente.

Dicho estudio, es una propuesta como ejemplo de trabajo en equipo con pertinencia social, puesto que se generó como producto de una demanda re- 
Revista de la Escuela de Ciencias de la Educación, año 10, número 9, enero a diciembre de 2014. Páginas 287-308. ISSN 1851-6297. ISSN En LINEA 2362-3349. RoL DE LA INVESTIGACIÓN EN EL DESARROLLO REGIONAL DEL NORTE DE LA PAZ. Miguel Yucra Rojas - Fredy EnRIQUe González.

gional y como un instrumento de planificación contemplando su ejecución con los futuros profesionales de la carrera de Ingeniería Industrial Amazónica con sede en San Buenaventura señalado anteriormente. Por lo tanto, como este ejemplo existen interesantes propuestas como oferta académica; sin embargo, la carencia de una vinculación institucionalizada entre la Universidad-Gobierno hace que se acumulen en las bibliotecas, cuando podría ser aplicado en dicha región.

\section{c) Necesidad de fortalecimiento tecnológico a la Carrera de Ingeniería Industrial Amazónica en San Buenaventura}

Las universidades bolivianas han creado programas de Postgrado en niveles M.Sc. y Ph.D., la mayoría lo ha hecho en áreas no tecnológicas (v.g. Educación Superior) que no aportan mucho a la creación de C\&T para el desarrollo socio-económico del país; son pocas las universidades que tienen programas de posgrado en las áreas tecnológicas y aún así adolecen de muchas fallas de organización y falta de profesores con niveles de Ph.D. (Escalera, 2005).

El año 2010 fue creada la carrera de Ingeniería Industrial Amazónica por la UMSA, en el municipio de San Buenaventura del departamento de La Paz, en el marco de la desconcentración universitaria, con la finalidad de dar respuesta a la demanda social y promover el desarrollo regional del norte de La Paz. Dichos estudiantes son originarios de dicha región, quienes en un periodo aproximado de dos años más podrán titularse como Ingenieros Industriales Amazónicos, donde la perspectiva de la formación está enfocada al desarrollo agroindustrial regional. Sin embargo, a fin de fortalecer dicha formación especializada; la UMSA deberá profundizar la práctica de investigación mediante el trabajo con docentes y estudiantes para promover la industrialización de los recursos naturales en coordinación con el municipio de la región amazónica y de esta manera proponer al gobierno departamental para su implementación; donde los profesionales titulados participen como gestores y la puesta en marcha de las iniciativas económicas.

De la modalidad anterior se podrían derivar dos beneficios: Uno la incorporación del profesional en su propia región y otro la capitalización de su propio desarrollo. Este escenario podría incrementar la visión de desarrollo regional, en vista que los futuros profesionales tendrían mayores oportunidades laborales al culminar su formación académica, inclusive de otras áreas de formación; que podría repercutir en el ejercicio profesional con visión investigativa y emprendedora para promover ciencia y tecnología. Asimismo, se estaría avanzando en el modelo de desarrollo desde la perspectiva del esquema de vinculación (esquema 2). Esto daría pie a que los trabajos de investigación a realizarse en la Universidad tengan un final de aplicación, y que la Educación Superior (pública y privada) se convierta en brazos de investigación y asesoramiento permanente para promover el desarrollo regional en coordinación con el Gobierno y los actores locales. 
Revista de la Escuela de Ciencias de la Educación, año 10, número 9, enero a diciembre de 2014. Páginas 287-308. ISSN 1851-6297. ISSN en LÍNEA 2362-3349. ROL DE LA INVESTIGACIÓN EN EL DESARROLLO REGIONAL DEL NORTE DE LA PAZ. Miguel Yucra Rojas - Fredy EnRIQue González.

Al respecto, con la finalidad de percibir la valoración de la investigación desde los estudiantes de último semestre, se consultaron a (50) estudiantes que experimentaron trabajos de investigación, con un rango de 23 a 25 años de edad, sobre la utilidad del trabajo de investigación; de los cuales el $84 \%$ de los estudiantes valoraron que la investigación es de mucha importancia para su formación profesional, por el contrario el $16 \%$ respondieron que les interesa poco o es indiferente la investigación.

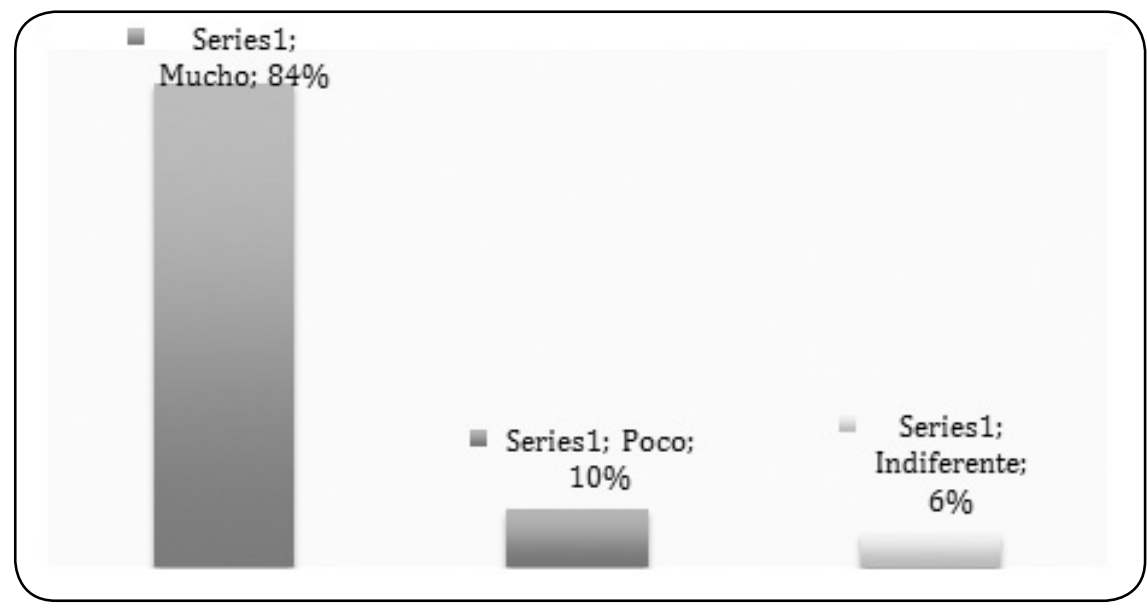

Gráfico 1: Utilidad del trabajo de investigación

De lo anterior se deduce dos escenarios; uno a aquella mayoría comprometido con su labor de proyecto de grado y la diferencia los indecisos en definir sus temas de investigación y los que aún no tenían en dicho momento sus temas respectivos. Por lo tanto, esta valoración estudiantil permite reforzar el planteamiento del modelo de desarrollo considerando la presencia del capital humano especializado con criterio de análisis e interpretación para coadyuvar la pertinencia de la investigación con la demanda social.

\section{d) Reorientación en el uso de los fondos IDH}

Por determinación del Honorable Consejo Universitario a partir del 2006, la UMSA aprueba la asignación del $12,5 \%$ de los Fondos del Impuesto Directo a los Hidrocarburos (I.D.H) anualmente, con el fin de fomentar el desarrollo de las actividades de investigación a través de Convocatorias a Fondos Concursables para la presentación de proyectos a través del Vicerrectorado y el Departamento de Investigación e Interacción Social (DIPGIS). Para el efecto se priorizaron las áreas de agrícola y pecuaria, tecnológica, económica, jurídica y social, salud, biodiversidad y cambio climático, tecnologías de información. 
Revista de la Escuela de Ciencias de la Educación, año 10, número 9, enero a diciembre de 2014. Páginas 287-308. ISSN 1851-6297. ISSN EN LINEA 2362-3349. RoL DE LA INVESTIGACIÓN EN EL DESARRolLO REGIONAL DEL NORTE DE LA PAZ. Miguel Yucra Rojas - Fredy EnRIQue González.

Durante el Simposio sobre Doctorado en Energía de la Universidad Mayor de San Simón, presentó una propuesta de distribución de los Fondos del Impuesto Directo a los Hidrocarburos (I.D.H) (Escalera, 2009).

- $\quad(20 \%)$ Financiamiento de proyectos y estudios de I\&D para atacar problemas básicos y estructurales regionales y darles soluciones a largo plazo.

- $\quad(20 \%)$ Financiamiento de Tesis de Postgrado, manejado por la Escuela de Postgrado de la UMSS.

Al respecto cabe destacar, una de las experiencias de la UMSA presentada el 27 de noviembre de 2012 en la ciudad de La Paz, de 26 proyectos ejecutados en un trabajo conjunto con docentes y estudiantes por las unidades académicas de la Facultad de Ingeniería, como producto del concurso de fondos provenientes del I.D.H., se pudo evidenciar que las iniciativas desarrolladas en un $11 \%$ tuvieron su origen de pertinencia a la demanda social con alguna región, el resto surgió de la iniciativa de los docentes o investigadores producto de sus experiencias profesionales. Esta diferencia de orientación de las investigaciones de pertinencia con la demanda local, generó repercusiones negativas en algunas instancias del gobierno central respecto al rol de la investigación desde la universidad.

Por lo tanto, se plantea que los recursos I.D.H. que la Universidad recibe del Gobierno Central, sean reformuladas para su utilización en función a la pertinencia con la demanda social en el marco del desarrollo regional y la política nacional del Estado Plurinacional de Bolivia; es decir reorientar la líneas de investigación priorizando el mapa de requerimiento de desarrollo regional, mediante el apoyo a la conclusión de estudios y tesis especializados en programas de pregrado y postgrado que permita la generación de proyectos productivos específicos con el asesoramiento directo de docentes. Por lo tanto, se deberá considerar la agenda nacional, regional y municipal para dicha reformulación de líneas de investigación.

\section{Conclusiones}

Para generar un cambio en el proceso de desarrollo regional del norte de La Paz, el modelo de desarrollo planteado y las cuatro acciones propuestas representan una oportunidad; lo contrario, significa seguir en un estancamiento respecto a la visión de la región amazónica y del país, que pueden ser traducidas en el estancamiento en la investigación, desperdicio de recursos provenientes de fondos financieros y consecuentemente postergada el desarrollo regional amazónico del norte de La Paz.

Las nuevas generaciones de profesionales deben formarse en escenarios coherentes a las realidades del país, considerando los nuevos modelos académicos y en coherencia a las exigencias de la demanda social y la globalización 
Revista de la Escuela de Ciencias de la Educación, año 10, número 9, enero a diciembre de 2014. Páginas 287-308. ISSN 1851-6297. ISSN en LÍNEA 2362-3349. ROL DE LA INVESTIGACIÓN EN EL DESARROLLO REGIONAL DEL NORTE DE LA PAZ. Miguel Yucra Rojas - Fredy EnRIQue González.

en educación superior; a partir de la cual se podría apalancar iniciativas de investigación acordes a la directriz nacional.

Las experiencias académicas presentadas son un ejemplo de caso relacionado con la formación profesional en un escenario de aprendizaje comunitario, como estos podrían realizarse con mayor profundidad, si logramos una interacción pertinente institucionalizada entre el actor, el facilitador y el que tiene el recurso financiero.

La experiencia profesional y la vivencia laboral en la región amazónica del norte de La Paz, con estudiantes, autoridades y población dedicada a actividades productivas; ha facilitado esta reflexión orientado a promover una vinculación institucionalizada, integración, participación proactiva y poner a disposición los recursos financieros, tecnológicos, de investigación e infraestructura tanto de la Universidad y el Gobierno; todo ello enlazado a una gestión de la investigación al interior de la UMSA, para atender las necesidades con pertinencia social a la región amazónica del norte de La Paz, Bolivia.

Como se puede observar, a pesar de contar con instrumentos normativos a nivel nacional, la presencia de la educación superior y los demandantes; no se ha logrado consolidar aun el enfoque presentado en los esquemas 1 y 2; y por lo tanto este escenario es posible que ocurran también en muchos países de Sudamérica, posiblemente debido a un aspecto común relacionado con la carencia de visión de país y una planificación justo para el periodo de turno, sea de la Universidad o del Gobierno.

\section{Referencias bibliográficas}

- Ley de la Educación "Avelino Siñani y Elizardo Pérez" de Bolivia (2010). Pág. 26-34.

- $\quad$ Austin, J. (1987). Guía Técnica de Agroindustria. Ecuador. Recuperado en wikipedia. org/wiki/Agroindustria.

- Asamblea General de Naciones Unidas (2000). Declaración del Milenio "Objetivos y Metas del Milenio". Resolución A/RES/55/2. New York, EE.UU.

- Declaración Universal de los Derechos Humanos (1948). New York.

- Escalera, S. J. (2002). Cómo generar Ciencia y Tecnología en Bolivia - Una Visión de compromiso social. Extracto Página 12. Cochabamba, Bolivia.

- Escalera, S. J. (2005). Análisis del Postgrado en las Universidades Bolivianas. Ponencia presentada en la 1er. Congreso de Educación Postgraduada en Sucre, Bolivia.

- Escalera, S. J. (2014). Cómo generar ciencia y tecnología en Bolivia - Una Visiona de compromiso social. Cochabamba, Bolivia.

- Gobierno Autónomo Departamental de La Paz (2013). "Pacto por La Paz" Diálogo Departamental.

- Hidalgo, A. (1998). El Pensamiento Económico sobre Desarrollo de los Mercantilistas. España.

- Nueva Constitución Política del Estado Plurinacional de Bolivia (2009). Pág. 26, 27,29 y 30 .

- Plan de Desarrollo del Departamento de La Paz al 2020 (2012). La Paz, Bolivia. 
Revista de la Escuela de Ciencias de la Educación, año 10, número 9, enero a diciembre de 2014. PÁginas 287-308. ISSN 1851-6297. ISSN EN LÍNEA 2362-3349. Rol DE LA INVESTIGACIÓN EN EL DESARROLLO REGIONAL DEL NORTE DE LA PAZ. Miguel Yucra Rojas - Fredy EnRIQUe GonzÁlez.

- Plan Nacional de Desarrollo (2006). La Paz, Bolivia.

- Plan Nacional de Ciencia, Tecnología e Innovación (2012). La Paz, Bolivia.

- $\quad$ PNUD (2001). Informe sobre el Índice de Desarrollo Humano de Bolivia. La Paz, Bolivia.

- Universidad Mayor de San Andrés (1988). Estatuto Orgánico. La Paz, Bolivia.

- $\quad$ Universidad Mayor de San Andrés (2012). Plan Estratégico Institucional 2012-2016. La Paz, Bolivia.

- Universidad Mayor de San Andrés (2013). V Convocatoria para la presentación de Fondos Concursables IDH 2013-2014. La Paz, Bolivia. 